\title{
L'Éthique du discours dans Wikipédia : la question de la neutralité dans une encyclopédie participative
}

Ethics of Discourse in Wikipedia: the Question of Neutrality in a Participative Encyclopedia

\section{Rivka Dvira}

\section{(2) OpenEdition Journals}

\section{Édition électronique}

URL : http://journals.openedition.org/aad/2286

DOI : 10.4000/aad. 2286

ISSN : 1565-8961

\section{Éditeur}

Université de Tel-Aviv

\section{Référence électronique}

Rivka Dvira, "L'Éthique du discours dans Wikipédia : la question de la neutralité dans une

encyclopédie participative », Argumentation et Analyse du Discours [En ligne], 17 | 2016, mis en ligne le 15 octobre 2016, consulté le 23 septembre 2019. URL : http://journals.openedition.org/aad/2286 ;

DOI : 10.4000/aad.2286

Ce document a été généré automatiquement le 23 septembre 2019.

\section{(c) $(1) \subseteq$}

Argumentation \& analyse du discours est mis à disposition selon les termes de la licence Creative Commons Attribution - Pas d'Utilisation Commerciale - Pas de Modification 4.0 International. 


\title{
L'Éthique du discours dans Wikipédia : la question de la neutralité dans une encyclopédie participative
}

\author{
Ethics of Discourse in Wikipedia: the Question of Neutrality in a Participative \\ Encyclopedia
}

Rivka Dvira

\section{Introduction}

1 L'encyclopédie virtuelle Wikipédia est devenue de nos jours une nouvelle «bible laïque ", comme l'était en son temps et l'est encore pour certains, le dictionnaire traditionnel. Nul ne peut nier la contribution de ce projet gratuit et collectif au monde de l'éducation, ni le fait qu'elle constitue aujourd'hui l'une des références essentielles de l'accès au savoir et de sa diffusion dans le monde informatisé. Sa spécificité réside dans le fait qu'elle est participative. Cette encyclopédie qui est nommée «l'encyclopédie libre » est en effet rédigée grâce à la coopération de tout individu considérant qu'il détient un savoir sur un sujet donné et qu'il est capable d'en rendre compte clairement. Le pouvoir de définir n'est donc plus l'apanage des institutions scientifiques.

2 L'attribution du droit à la parole à des internautes qui ne sont pas forcément des experts lexicographes aurait pu entraîner un renoncement au critère de l'objectivité discursive. Or, il n'en est rien. Wikipédia, aussi révolutionnaire soit-elle, accorde une place centrale à la «Neutralité du point de vue» dans ses principes fondateurs. Ce principe, qui atteste de l'aspiration à présenter de l'information fiable, exige une construction du savoir dépourvue de tout biais idéologique. Il demande une suspension de l'acte de juger au profit d'une présentation de tous les points de vue concernant 
l'objet de l'entrée définie. Or, comme l'a bien montré Emile Benveniste dans Problèmes de linguistique générale (1966), le système du langage est intrinsèquement subjectif. La prétention de ce nouveau type de média à la neutralité parait donc comme plus paradoxale encore qu'à première vue.

3 On tentera ici de revisiter la question de la non-intervention discursive au prisme de cette révolution culturelle que constitue la rédaction "participative» de Wikipédia. Que gagne le savoir et qu'en est-il du concept de «neutralité » dans un univers discursif où le consensus entre les rédacteurs est exigé pour considérer une entrée comme neutre, et où la responsabilité éthique du contenu repose sur un groupe? Après une brève mise au point sur les techniques de travail de Wikipédia, on analysera la façon dont la notion de neutralité est définie dans le cadre du site, et on livrera les notions et définitions élaborées par Wikipédia à une analyse critique fondée sur les théories de l'analyse du discours. On présentera ensuite l'analyse d'un extrait d'une entrée de Wikipédia et de la discussion des contributeurs qui l'accompagne. Cet exemple restreint, représentatif d'un corpus beaucoup plus vaste ${ }^{1}$, permet d'examiner des sujets éthiques primordiaux : la conception de la construction du savoir et son rapport avec le jugement de valeur, et la responsabilité du lexicographe (en l'occurrence tout un chacun) quant à la présentation équilibrée des points de vue. En somme, les questions soulevées sont: le concept de "neutralité » subit-il des modifications dans l'espace discursif novateur que propose Wikipédia? Quelles sont de facto les valeurs qui guident les contributeurs vers la qualification d'une entrée comme « neutre»?

\section{Brève mise au point sur les techniques de travail de Wikipédia}

4 Le site Wikipédia, qui émerge sur la toile en 2001, continue la tradition des encyclopédies classiques aspirant à englober la totalité du savoir humain et à le mettre à la disposition de chacun ${ }^{2}$. Les changements technologiques que permet l'usage d'internet semblent fournir une plate-forme qui convient parfaitement à cette ambition. Les sites Wiki ont comme spécificité le fait que leurs pages peuvent être modifiées par les visiteurs du site. L'idée d'établir un projet d'encyclopédie libre et multilingue à l'aide de cette technique permet non seulement une diffusion des plus populaires au monde, mais aussi une transformation de la manière dont le savoir est construit. La conception selon laquelle un expert élu et érudit partage le savoir dont il est propriétaire avec le peuple ignorant change : dans Wikipédia chacun détient une part du savoir, et le partage avec ses égaux. Une relation horizontale entre rédacteur et lecteur et entre lecteur et savoir est alors établie. Le savoir subit un processus de démocratisation. Sans autorité supérieure qui pilote l'évolution et la construction du savoir, Wikipédia représente un modèle décentralisé et communautaire, dont la gestion est basée sur le commun accord des contributeurs.

5 Le travail collectif qui a pour but d'établir une définition complète d'un terme diffère lui aussi de la technique à la base de l'encyclopédie classique, dans laquelle un seul expert rédige une entrée entière ${ }^{3}$. Le travail commun sur la rédaction d'une entrée, dont la mise en mots reste rarement statique et peut être revue ad infinitum, s'effectue grâce au système de pages de discussions qui accompagnent l'entrée et qui sont accessible à tout lecteur qui s'intéresse au processus de rédaction. C'est dans ces pages 
de discussions que les contributeurs peuvent débattre et expliciter leurs points de vue personnels.

6 Ces changements fondamentaux exigent un système de règles et de principes qui gèrent et orientent le travail. Il existe en effet cinq «principes fondateurs de Wikipédia» qui représentent les piliers de la réglementation. Le principe de «Pertinence encyclopédique » établit l'identité du site en tant qu'encyclopédie. Il définit le sens et le but d'une encyclopédie en général, de Wikipédia en particulier, et précise ce que le site ne souhaite pas être. Wikipédia espère ainsi éviter les types d'interventions partisanes que sa nature participative pourrait entraîner. Le principe de la "publication sous licence libre » donne le droit à chacun d'utiliser les textes de définitions publiées dans Wikipédia sans limitation, mais en prenant garde de mentionner qu'ils appartiennent au site. Le principe nommé « Savoir-vivre " gère les recommandations de politesse et de civilité qui font partie de l'espace discursif de la communauté Wikipédia. Le principe concernant la « Flexibilité des règles » définit qu'il n'existe aucune règle fixe hormis les cinq principes fondateurs. La responsabilité concernant la réglementation du travail est donc assumée non par une instance extérieure à la rédaction, mais par la communauté des contributeurs.

\section{Mise en mots du principe de neutralité de Wikipédia}

7 Le principe fondateur qui concerne les questions fondamentales de cet article est celui qui est nommé la " Neutralité du point de vue » : l'encyclopédie pose comme condition de rédaction une présentation équilibrée de tous les points de vue qui existent à propos de l'objet de l'article rédigé, sans en favoriser aucun ${ }^{4}$. Notre objectif est ici de faire le point sur la façon dont Wikipédia définit la notion de neutralité, en exposant les difficultés rencontrées et en signalant dans quelle mesure cette définition est en accord avec les théories de l'analyse du discours et sa conception de la subjectivité dans le langage. Précisons que par "subjectivité » dans le contexte de cet article, nous nous référons aux dimensions affective, évaluative et axiologique de la subjectivité, donc à la prise de position implicite ou explicite (et non à la dimension déictique de la subjectivité dans le langage). Il s'agira ensuite de vérifier dans quelle mesure Wikipédia respecte dans sa pratique le principe de neutralité qu'elle a elle-même défini.

8 La définition du principe de neutralité de point de vue est composée de deux approches : une approche positive et une approche négative. L'approche positive a pour but d'expliciter ce qu'est la neutralité dans ce contexte et ce qu'il faut faire pour l'atteindre. L'approche négative précise «ce que la neutralité n'est pas » et les fautes à éviter si l'on veut rester neutre.

$9 \quad$ L'approche positive est formulée ainsi :

[La neutralité de point de vue] suppose de décrire plusieurs points de vue; de représenter chacun de ces points de vue aussi fidèlement que possible, en tenant compte de leurs importances respectives dans le champ des savoirs; de fournir le contexte nécessaire à la compréhension de ces points de vue ainsi que de qui les tient ("Wikipédia : Principes fondateurs »).

10 La définition "décrire plusieurs points de vue » souligne le côté pluraliste de la neutralité. L'exigence "Aussi fidèlement que possible» dévoile l'aspiration à l'adéquation de la mise en mots à la réalité du référent, dans laquelle l'encyclopédie voit la marque de l'«objectivité $»^{5}$. Il s'agit pour Wikipédia de garantir une 
correspondance exhaustive entre la mise en mots et le référent qu'elle transcrit. L'exigence de représenter les points de vue selon leurs "degrés d'importance », soit présenter les différentes opinions de manière à ce que chaque opinion soit présentée dans l'entrée en fonction de son importance dans les champs du savoir (de par leur longueur de rédaction ou leur ordre de présentation) renforce l'aspiration à la « fidélité » exprimée plus haut. La rédaction "fidèle » ne serait donc pas seulement, d'après Wikipédia, le résultat d'une description exacte mais résulterait aussi d'une organisation du texte susceptible de représenter la "réalité telle quelle». Or, cette exigence nécessite malgré tout de la part du rédacteur une procédure de hiérarchisation, ou d'évaluation d'une hiérarchie intrinsèque, inhérente au point de vue à représenter. Cette exigence de la part des fondateurs de Wikipédia nous paraît donc en contradiction avec la définition négative de la neutralité qui sera présentée dans le développement qui suit, définition qui veut que l'entrée ne contienne aucune évaluation personnelle du rédacteur.

11 L'exigence de «Fournir le contexte nécessaire à la compréhension de ces points de vue» confirme la pertinence d'un des axiomes des linguistiques pragmatique et énonciative contemporaines comme de la nouvelle rhétorique perelmanienne: le contexte est une composante intrinsèque de la construction du sens. "Ainsi que de qui les tient » réfère à l'argument d'autorité, au statut social, à l'ethos préalable, bref à la situation d'énonciation socio-historique configurant l'identité du locuteur. Cette exigence de «fournir le contexte » de l'information décrite dans l'entrée est étayée par le devoir de "sourcer » chaque affirmation qui pourrait poser problème ou causer un conflit entre les contributeurs. "Sourcer » signifie ici procurer une source extérieure à Wikipédia qui confirme telle ou telle assertion dans le contexte de la définition. Les règles de Wikipédia concernant la neutralité imposent de fournir une source de référence pour chacun des points de vue présentés dans la définition et de citer leurs partisans, afin que les points de vue ne soient pas représentés comme appartenant aux rédacteurs. Cette pratique académique, utilisée initialement par l'historien Leopold von Ranke au début du $19^{\mathrm{e}}$ siècle, est censée garantir la légitimité et la neutralité du savoir partagé dans le site (Langlais $2015: 80$ ).

Dans ce cadre, rien de plus "objectif " à première vue que la citation. Cependant, comme le montrent de nombreux analystes du discours ${ }^{6}$, cette conviction est problématique pour plusieurs raisons: la sélection subjective de la citation par le contributeur, la reformulation de la citation en cas de discours indirect rapporté, le choix du co-texte de la citation par le locuteur et enfin, le détachement de la citation de son contexte d'énonciation. Là encore, l'effacement total de la subjectivité s'avère impossible.

13 L'approche négative est explicitée par cette définition : « ne représenter aucun point de vue comme étant la vérité ou le meilleur point de vue» ("Wikipédia: Principes fondateurs»). La définition marque une rupture entre «le point de vue» et «la vérité ». Représenter un point de vue comme étant la vérité implique l'usage dans l'énoncé d'« effets d'objectivité » (Koren 1996). D'après cette définition, la rédaction devrait donc éviter cette pratique linguistique. De plus, cette directive vise à mettre en garde les rédacteurs contre la conviction de pouvoir détenir la Vérité absolue. D’autre part, cette définition souligne le devoir du rédacteur de s'éloigner de toute évaluation des points de vue qu'il décrit ou d'établir la moindre hiérarchie entre son propre point de vue et celui des autres. Représenter un point de vue comme étant le meilleur 
suppose une évocation plus ou moins implicite du savoir du rédacteur, mais aussi de ses goûts, de ses valeurs et de ses opinions. Pour que le texte soit pluraliste, la rédaction devra éviter tout signe linguistique qui sous-entendrait une préférence ou une objection du rédacteur vis-à-vis du point de vue présenté7. La neutralité se traduirait donc dans le contexte de Wikipédia comme une absence de prise de position - explicite ou implicite - par le contributeur.

Or, le savoir acquis à ce jour dans les sciences du langage permet de penser qu'il s'agit là d'un mythe inspiré par une conception rationaliste du langage, comme le démontre Koren à propos du journalisme (ibid.). Cette conception ne tient pas compte des acquis de la linguistique moderne. Celle-ci a en effet établi depuis Saussure qu'il n'existe pas de locuteur absent de ses propres dires : tout énoncé résulte d'une série de sélections et de décisions impliquant nécessairement une prise de position. Rappelons ici l'affirmation de Kerbrat-Orecchioni (2009 [1980] : 79) : « toute unité lexicale est, en un sens, subjective, puisque les 'mots' de la langue ne sont jamais que des symboles substitutifs et interprétatifs des 'choses' ».

Le site Wikipédia veille également à marquer un écart entre "neutralité » et « objectivité »:

The NPOV policy says nothing about objectivity. In particular, the policy does not say that there is such a thing as objectivity in a philosophical sense-a "view from nowhere" [...]. That is not the policy, and it is not our aim! Rather, to be neutral is to describe debates rather than engage in them ( $« \mathrm{FAQ}$, Wikipedia en anglais: There's no such thing as objectivity ", consulté le 13-06-2011).

L'objectivité est donc considérée comme une manière de décrire la réalité « de nulle part ", c'est-à-dire sans la moindre intervention d'un locuteur absent de ses propres dires. L'objectivité concerne la qualité de la description des réalités extralinguistiques, description qui doit être fidèle et exhaustive. Par contre, la neutralité renvoie à la qualité de la présentation des points de vue dans le cas d'une polémique, à leur présentation égalitaire impliquant la suspension de l'acte d'évaluer ou de juger. Une analyse approfondie des définitions de "neutralité » et d'«objectivité » dans les dictionnaires lexicographiques révèle en effet une différence de sens entre ces deux termes, souvent confondus dans le discours ordinaire: le locuteur dit "neutre» participerait à une polémique sans se prononcer en faveur de l'un des camps. Le locuteur objectif, lui, se trouve dans une situation où il doit décrire une réalité de l'extérieur, sans énoncer ses goûts ou ses dispositions vis-à-vis de l'objet qu'il décrit. De même, la théorie de l'argumentation de Chaïm Perelman, à la différence de la conception cartésienne de la rationalité, distingue entre «impartialité » et « objectivité » (2008 [1958] : 78-79). L'impartialité du tiers est la qualité l'autorisant à remplir ultérieurement la fonction de juge. Comme tout être humain solidaire d'un groupe social, il ne peut faire abstraction des convictions et des valeurs de ce groupe. Il ne peut donc pas être objectif, soit entièrement étranger aux valeurs du groupe au moment de trancher en faveur du point de vue le plus pertinent à ses yeux. Etre impartial, c'est être équitable, entendu comme à l'écoute de tous les points de vue dans un premier temps heuristique de suspension du jugement ; cette impartialité débouche non pas sur l'objectivité d'un jugement de fait, mais sur l'acte de trancher en faveur de l'un des points de vue en connaissance de cause. Dans le contexte de Wikipédia, il est exigé de la part des contributeurs d'être impartiaux, de ne pas trancher en faveur de l'un des camps. 

l'intersubjectivité comme condition de neutralité : Jimbo Wales définit le point de vue neutre en ces termes : « Le point de vue neutre consiste à essayer de présenter les idées et les faits de façon à ce que les partisans et les détracteurs puissent s'accorder. Bien sûr, un accord à $100 \%$ est impossible " (Wales, "Wikipédia : Neutralité de point de vue »). degré d'adéquation de la définition à la réalité, mais plutôt le degré d'accord entre les éditeurs. La neutralité pour Wales serait ainsi liée aux notions d'interdiscours et d'intersubjectivité, au sens de Benveniste dans Problèmes de linguistique générale, à savoir la co-construction du sens linguistique par plusieurs interlocuteurs ${ }^{8}$. Il existe aussi un rapport entre cette conception de la neutralité et la définition extrinsèque du fait proposée par Perelman dans la Nouvelle rhétorique ${ }^{9}$. Un fait, dans les interactions argumentatives, n'aurait pas de définition intrinsèque a priori et serait établi a posteriori par l'établissement d'un consensus, au terme d'une négociation entre les proposants et les opposants.

19 C'est précisément cette conception participative de la rédaction qui représente la nouveauté de Wikipédia par rapport aux encyclopédies classiques : la représentation adéquate de la réalité doit être le fruit d'un travail collectif de discussion et de concertation argumentées, alors que dans les encyclopédies classiques, l'autorité et la responsabilité éthique du contenu équilibré et adéquat sont celles d'un ou de quelques rédacteurs professionnels. La responsabilité de la vérité référentielle et l'éthique de l'acte de dire ne sont plus assumées par une seule personne, mais par un groupe.

Pour résumer la conception de Wikipédia de la neutralité, cette encyclopédie moderne adhère au devoir de neutralité d'après les définitions de ce concept par les encyclopédies classiques ${ }^{10}$, mais avec deux précisions. D'une part, la déclaration de Wikipédia concernant la neutralité est focalisée sur la description neutre de débats concernant les sujets des entrées et pas forcément sur la description objective des faits. D'autre part, la qualification d'une entrée comme neutre devrait résulter non seulement du travail collectif d'un groupe de contributeurs, mais aussi d'un commun accord de ceux-ci concernant cette qualification. Cette conception est soutenue par la gestion participative du site, laissant la principale responsabilité de la rédaction et de l'évaluation de la neutralité aux participants eux-mêmes sans qu'il n'y ait aucune instance officielle qui supervise les résultats des actes d'évaluation.

21 La notion de «neutralité » wikipédienne, comme nous l'avons montré, s'avère avoir plusieurs points communs avec la notion d'«objectivité », du point de vue de la pratique de l'éditeur : l'effacement énonciatif, l'idéalisation de la citation et l'aspiration à l'exhaustivité. Il y a donc dans les consignes de Wikipédia une part d'objectivisme qui est en contradiction avec le savoir acquis dans le domaine de la linguistique de l'énonciation. Dans l'analyse qui suit nous montrerons comment ces consignes sont appliquées et nous signalerons les cas où la subjectivité du locuteur et ses prises de position se manifestent malgré les instructions du site. 


\section{Analyse de l'introduction de l'entrée « Lehi »}

Que deviennent ces principes dans le cadre de la rédaction des articles de Wikipédia? On présentera ici, afin de tenter de répondre à cette question, l'analyse de l'introduction de l'entrée "Lehi $»^{11}$ de la version francophone du site, ainsi que l'analyse de la discussion qui l'accompagne. Ce passage nous paraît particulièrement représentatif de la manière dont le processus de rédaction influence ce qui est affiché dans l'entrée. Il illustre par ailleurs les cas où de manière surprenante, une entrée qui contient des marques de subjectivité qui indiquent une prise de position obtient la qualification «article de qualité » de la part des contributeurs, ce qui implique que la majorité sinon la totalité des contributeurs considèrent qu'elle est " neutre ». L'analyse interroge le degré de conformité de la rédaction de facto avec les règles de l'encyclopédiie ${ }^{12}$.

L'entrée « Lehi $»^{13}$ a été proclamée « article de qualité » au terme d'un tour de vote dans lequel $90 \%$ des contributeurs ${ }^{14}$ ont opté pour cette mention, en octobre 2006. La légitimité des actes du Lehi a suscité à l'époque de son existence, et suscite encore aujourd'hui, une controverse concernant la légitimité de la violence de ses actes. Il est donc compréhensible qu'un contributeur considère ce sujet comme particulièrement difficile à traiter de manière neutre : «c'était une gageure et on est très agréablement surpris de la densité et de la neutralité de l'information", dit-il. Les résultats de l'analyse linguistique et discursive "au ras des mots" de l'introduction de l'entrée remettent pourtant en cause les décisions des contributeurs concernant la neutralité du texte. On tentera de le démontrer dans nos commentaires concernant ce passage. Afin d'assurer l'impartialité descriptive exigée par le chercheur, nous adoptons la solution de Plantin (2002: 234): nous confronterons discours (l'introduction en français) et contre-discours (les introductions en anglais et en hébreu), ce qui nous permettra de décrire et d'analyser le passage en question avec le maximum de distance. Mais l'impartialité, comme le dit Perelman, n'est qu'une étape heuristique de la recherche. Mon ${ }^{15}$ devoir est ensuite de trancher et de montrer où les différences entre les diverses versions peuvent avoir des conséquences problématiques, ce que je tâcherai de faire.

Catherine Kerbrat-Orecchioni (2009 [1980]) recense et définit cinq types différents d'interventions subjectives. Ces types d'interventions sont l'intervention par sélection, l'intervention par organisation hiérarchique des informations, la subjectivité de type «interprétatif», la subjectivité modalisatrice et enfin la subjectivité axiologique ${ }^{16}$. L'analyse approfondie de l'introduction de l'entrée révèle la présence des cinq types d'interventions. Il ne sera cependant possible, faute de place, de signaler ici que l'un des aspects de l'intervention subjective qui véhicule une prise de position. On présentera donc l'aspect qui est l'objet de la controverse dans l'extrait de la discussion entre les contributeurs présenté par la suite, pour donner une vue d'ensemble du processus d'analyse auquel nous avons procédé. Le type d'intervention subjective retenu ici est l'intervention par sélection.

Il s'agit pour le locuteur de choisir parmi la masse des détails qui constituent l'événement ou le sujet de la description, les faits qui méritent d'être verbalisés. Wikipédia comme toute autre encyclopédie exprime un désir d'exhaustivité. Or, comme le souligne Koren (1996: 39-42), l'exhaustivité est inapplicable à la lettre, puisque la sélection des informations pertinentes est une obligation linguistique incontournable, 
imposée par la nature du langage et des lois du discours. L'exhaustivité ne signifie pas «tout dire sur tout», mais dire seulement tout ce qui, dans une situation donnée et compte tenu du savoir préalable de l'énonciateur et de ses co-locuteurs, est pertinent sur un sujet donné. Il est donc inévitable pour le locuteur de choisir et il exercera ce choix dans la plupart des cas en fonction de son savoir, mais aussi de ses convictions, de ses croyances et de son point de vue.

La sélection dans cette introduction concerne le choix des faits mentionnés dans la définition. Dans le deuxième paragraphe (L. 9-13), il est question des relations idéologiques et pratiques du Lehi avec les régimes totalitaires de l'époque : le Nazisme allemand et le Fascisme italien. La mention de ces notions politiques, porteuses de connotations péjoratives dans la doxa contemporaine, dans le contexte d'une introduction sur le Lehi, sans la moindre distance critique, risque de conduire à une prise de position implicite, à savoir l'établissement d'un lien important entre le Lehi et ces courants idéologiques: "Dans son combat contre les Britanniques, le groupe tentera sans succès des contacts en 1941 avec les Italiens et avec les autorités nazies » (L. 9-10). Dire que le Lehi a voulu être en contact pour des raisons politiques avec des régimes totalitaires et considérés comme meurtriers, peut sous-entendre une proximité idéologique des premiers avec les idées des seconds. Ces faits ne sont démentis par aucune source concernant le Lehi. Si ces informations correspondent à des faits historiques exacts et avérés, les signaler est légitime. Pourtant, dans le contexte présent, des questions de fond mériteraient d'être soulevées, comme le prouve l'analyse des introductions parallèles en anglais et en hébreu.

Celle que nous traiterons ici concerne l'importance conférée dans l'introduction de l'entrée à cette information. La comparaison avec un extrait de l'entrée anglophone sur le Lehi, concernant spécifiquement les relations du Lehi avec le nazisme peut fournir une certaine clarification sur ce point.

Extrait de l'introduction de l'entrée en anglais

Lehi initially sought an alliance with Fascist Italy and Nazi Germany, offering to fight alongside them against the British in return for the transfer of all Jews from Nazi-occupied Europe to Palestine. On the belief that Nazi Germany was a lesser enemy of the Jews than Britain, Lehi twice attempted to form an alliance with the Nazis.

Dans ce passage, le rédacteur mentionne la relation établie entre le Lehi et le régime nazi. On peut relever cependant une différence essentielle: le fait est accompagné d'une explication levant immédiatement tout risque d'ambiguïté. Les raisons pour lesquelles le Lehi contacte les Nazis sont prises en compte et dûment exposées : il s'agit de combattre la politique de restriction de l'immigration juive des Britanniques, afin de sauver les Juifs d'Europe en les transférant en Palestine.

L'extrait équivalent issu de l'entrée en hébreu fournit une précision supplémentaire concernant les relations entre le Lehi et le régime Nazi et concernant les intentions politiques qui ont amenées à ces actes :

31 Extrait de l'introduction de l'entrée en hébreu

[En 1940, l'organisation a essayé de signer un pacte avec le régime nazi en Allemagne afin de sauver la plupart des Juifs européens du danger d'extermination et de les faire immigrer en Eretz-Israël. En récompense le Lehi promit de combattre l'empire britannique de l'intérieur d'Eretz-Israël.] (Je traduis) 
Deux lexèmes dans cette présentation des faits influencent profondément le point de vue qui émane de la mise en mots : premièrement le nom [iska], pacte : dans l'espace politique, l'histoire le prouve bien, les pactes entre ennemis sont envisageables. A la différence d'«alliance", utilisé dans l'introduction en anglais, qui suggère une proximité idéologique entre les deux institutions, "pacte » réfère à un accord basé sur des intérêts, sans impliquer une vision du monde commune. D'autre part, le verbe [lehatsil], sauver, suggère l'urgence d'un tel pacte : tous les moyens seraient légitimes dès lors qu'il s'agit des sauver des vies. La relation Lehi-Nazisme ne serait donc pas idéologique d'après cette présentation, mais justifiée par une question de vie et de mort.

33 Il est donc possible de déduire à partir de la lecture de l'entrée anglophone et de l'entrée en hébreu, que les relations du Lehi avec le Nazisme étaient bien plus complexes que ce qui ressort de la description de l'entrée francophone à ce sujet. L'introduction qui a pour but de résumer pour le lecteur l'essence du sujet et qui représente en quelque sorte le contenu et l'esprit de l'entrée entière contribue donc, dans l'introduction en français, à créer un malentendu. Il semble que l'établissement de ce lien dans l'introduction aurait une autre visée que le simple résumé: attirer l'attention par l'intermédiaire de faits "sensationnels » sur une alliance contre-nature entre des Juifs vivant en Israël et le régime nazi. Sans se prononcer forcément pour ou contre cette décision du Lehi, il est certain que ce type de décontextualisation risque d'induire le lecteur ordinaire en erreur et de disqualifier d'emblée le Lehi et ses actes.

34 Le risque de malentendu est d'ailleurs confirmé par le dialogue suivant, extrait du site «Yahoo! Questions réponses » qui sert d'« espace où l'on peut poser des questions et recevoir gratuitement des réponses d'autres internautes dans tous les domaines ${ }^{17}$. Les réponses aux questions posées par les internautes ne sont pas forcément données par des experts. L'un des contributeurs de ce site publie cette question : "Connaissez-vous le Lehi, groupe armé sioniste pronazi et pro fasciste?». Ce contributeur (anonyme) joint à cette question l'introduction de l'entrée «Lehi » de Wikipédia. Deux réponses d'internautes à propos de cette question méritent d'être mentionnées. La première, de l'internaute surnommée 'Fifille' : "J'hallucine, des juifs nazis????, Morte de rire! ». La deuxième, de l'internaute 'echoesfunder': "Ah..! Bon c'est nouveau. Qu'ils s'entre éliminent et ne font pas chier le monde ces cafards. ».

Ces trois interventions renforcent l'hypothèse que la mise en mots de cette partie de l'introduction peut causer une interprétation biaisée de l'idéologie du Lehi ${ }^{18}$. Premièrement, la question présentée par le contributeur anonyme comporte un présupposé qui consiste en la juxtaposition des adjectifs "Sioniste », "pro-nazi » et "pro-fasciste ». L'internaute prendrait pour acquise l'identité "pro-nazie » du Lehi suite à la rencontre mentionnée dans l'introduction de l'entrée Wikipédia. Cette présupposition va plus loin encore dans la remarque de 'Fifille' : « des juifs nazis ??? », appellation qui lui semble surprenante (les exclamations "J'hallucine», "Morte de rire " témoignent de cette surprise). Ils ne seraient donc plus "pronazis» (qui soutiennent les idées des Nazis) mais carrément nazis eux-mêmes. Dans la troisième remarque, celle de 'echoesfunder', ce n'est plus d'une qualification qu'il est question, mais d'une déduction «logique » qui découlerait de cette dénomination paradoxale : s'ils sont Juifs d'une part et nazis (célèbres pour leur projet d'éliminer le peuple juif) d'autre part, alors il en résulte qu'ils s'entre-éliminent ou s'auto-éliminent. La démarche «logique » que font ces internautes serait donc: «Le Lehi a rencontré les 
Nazis donc Le Lehi est pronazi donc Le Lehi est Nazi donc Le Lehi aspire à tuer les membres de son propre peuple».

Kerbrat-Orecchioni (1981:52-53) mentionne dans son chapitre Argumentation et mauvaise foi ce genre de raisonnement comme étant un des types d'argumentation fallacieuse qui peuvent être analysés par des outils linguistiques. Il s'agit d'un des cas de la "généralisation abusive ", qui consiste à déduire de « $X$ et $Y$ se rencontrent » que " $\mathrm{X}$ et $\mathrm{Y}$ c'est la même chose ». Le simple fait de mentionner dans l'introduction de l'entrée la rencontre du Lehi avec les représentants du gouvernement nazi ne représente pas encore ce genre de saut argumentatif. Pourtant, comme en témoigne la discussion dans le forum du site "Yahoo!», cette démarche fallacieuse risque de se produire quand ce fait est mentionné sans contexte. Je présume que les rédacteurs d'une entrée encyclopédique détiennent une part de responsabilité éthique concernant les interprétations possibles de leur texte. Si les lecteurs du site « Yahoo réponses » ont interprété les choses d'une telle manière, c'est qu'il y avait dans le texte une pratique discursive qui le permettait, même si ce n'était pas forcément l'intention des rédacteurs a priori.

L'introduction de l'entrée «Lehi » précède une entrée considérée comme «neutre » par la communauté Wikipédia. Pourtant, l'analyse révèle l'existence de plusieurs types d'interventions subjectives dans le texte, dont l'intervention par sélection, présentée ci-dessus. Ces interventions offrent une image critique dévalorisante de l'organisation, confortant l'opinion selon laquelle le Lehi représentait une organisation dont les actes et les choix étaient illégitimes. Toutefois, en réalité, cette mise en mots ne représente qu'un point de vue. Comme déjà précisé, le Lehi est sujet de controverse entre ceux qui le critiquent et ceux qui le considèrent comme un mouvement de résistance « combattant pour la libération d'Israël » et non comme une organisation terroriste au sens actuel du terme. Or, les règles de neutralité de Wikipédia veulent qu'à partir du moment où il existe plus d'un point de vue sur le sujet de l'entrée, tous les points de vue doivent être explicités impartialement. La subjectivité de cet extrait se situe donc dans la transgression de la règle de pluralisme des points de vue, mais sans que cela n'ait affecté le choix de la mention « article de qualité ».

Comme signalé plus haut, ce qui est surprenant n'est pas le fait qu'il existe de la subjectivité dans ce passage, mais plutôt le vote unanime des contributeurs en faveur de la mention 'article de qualité'. Ce vote signifie que pour eux la neutralité de cette entrée va de soi. On tentera d'élucider ce paradoxe dans le développement suivant.

\section{Analyse de la discussion de l'entrée « Lehi »}

L'extrait de discussion présenté ici traite essentiellement d'une version archivée de l'introduction de l'entrée «Lehi » qui date du 11 Août 2006, deux mois avant le vote pour la mention de l'article "article de qualité $»^{19}$. On analysera ici le processus de neutralisation lors d'un désaccord concernant la neutralité.

On aura recours aux observations d'Amossy (2011: 25-42) au sujet du discours polémique et à la conception de l'argumentation de Perelman et Olbrechts-Tyteca (2008 [1958]) pour éclaircir les résultats de l'analyse de la dynamique des discussions. Pour Amossy, si le but d'une argumentation est de trouver la réponse à une question éthique ou pratique, il existe néanmoins un continuum à deux extrêmes sur lequel peuvent être placés les différents types de discussions (2011:27). A l'une des extrémités du 
continuum se trouve un pôle qu'Amossy nomme la "co-construction des réponses ", c'est-à-dire un processus coopératif de tous les protagonistes qui a pour but d'arriver à une réponse commune à la question posée. Ce pôle est défini par les caractéristiques suivantes : premièrement l'écoute de l'auditoire et la construction d'un discours qui prend en compte les sensibilités de celui-ci. Ensuite, une attitude du locuteur qui accepte l'éventualité d'une erreur de sa part et le devoir de justifier ses prises de positions, puis enfin l'allusion à des arguments admis comme valables pour l'humanité entière, acceptés comme Vérité, et la référence à l'idéal du consensus (Perelman et Olbrechts-Tyteca 2008). Le deuxième pôle de ce continuum est la "confrontation violente des thèses antagonistes", à savoir la polémique. Ce pôle est caractérisé par une opposition des discours, par un degré élevé d'intertextualité et de dialogisme, par une disqualification de l'autre point de vue et/ou de l'autre participant et par la prise à témoin d'un tiers ou d'un public.

41 C'est du premier pôle, on le verra, que relève la discussion de l'entrée Lehi dans Wikipedia. La conversation entre les deux contributeurs dans l'extrait de la discussion se déroule en effet dans la convivialité et la bonne entente qui sont caractérisées par plusieurs traits: l'argumentation justifiée, la recherche de l'accord du co-locuteur, l'humilité et l'ouverture à la critique.

\section{Argumentation justifiée}

Les contributeurs veillent à donner des arguments pour chaque critique qu'ils avancent du contenu de l'entrée. Par exemple, 'Cristophe Cagé' qui ouvre la discussion explique le fait de retirer les modifications de 'Freeman2006' (L.2) : « car non sourcées, hors sujet ou non neutres. ». La conjonction «car » introduit la justification de l'énoncé qui la précède.

\section{Recherche de l'accord du co-locuteur}

43 Les changements que proposent les contributeurs dans le contenu de l'entrée à partir du moment où la discussion est lancée ne sont envisagés qu'à condition que l'autre y consente : 'Christophe Cagé' dit (L. 36-38) «Je peut (sic) faire cette modif [modification], si nous sommes d'accord» et 'Freeman2006' dit (L. 40-41) « si vous êtes d'accord alors dites le moi ».

\section{Humilité et ouverture à la critique}

Ce qui contribue peut-être le plus au caractère convivial de cette discussion est le fait que les contributeurs font preuve d'une ouverture à la critique et à la possibilité qu'ils ne détiennent pas la vérité $\mathrm{V}$ : 'Freeman2006' insiste sur le fait qu'il est nouveau sur le site, ce qui lui sert d'explication des erreurs potentielles de rédaction de sa part (L. 20): «En fait je suis nouveau sur ce site et l'introduction que j'ai mis ce n'est que la copie de l'article etc... ", Les deux contributeurs affirment clairement à plusieurs reprises qu'ils sont ouverts à toute critique 'Christophe Cagé' (L. 5) : «Je reste ouvert à la preuve contraire, mais je suis dubitatif » et 'Freeman2006' (L. 32-33) «Si vous avez d'autres critiques, alors n'hésitez pas à me le dire ».

En somme, l'analyse de la discussion concernant l'entrée «Lehi» montre que le caractère convivial de la discussion conduit à un accord qui se traduit finalement par 
un vote unanime en faveur de la catégorisation " article de qualité ». Comme on a tenté de le démontrer plus haut, le processus de neutralisation qui se déroule dans cette page de discussion ne parvient pas à créer une introduction neutre d'après les règles de Wikipédia. Ce qui finit par être considéré comme une vérité qui va de soi par les contributeurs de l'article en français à cette date-là se révèle être problématique d'après les observations exposées dans le cadre de l'analyse de l'entrée et au prisme des entrées en anglais et en hébreu. Nos observations concernant l'entrée "Lehi» démontrent que de facto les contributeurs de l'entrée mettent le principe de "Savoirvivre $»^{20}$ et l'idée de consensus au-dessus du devoir de renoncement au point de vue subjectif, et donc du devoir de neutralité et de suspension du jugement.

\section{Conclusion}

La neutralité wikipédienne veut que dans le cas où, concernant une entrée, il existe plus d'une opinion, tous les points de vue doivent être mentionnés de manière équilibrée. En tenant compte du fait que l'effacement de la subjectivité dans le langage est nécessairement illusoire, on peut se demander quel est le processus qui permet aux contributeurs de qualifier certaines entrées de neutres et d'autres de non-neutres, là où l'analyse des pratiques discursives ne dévoile aucune différence primordiale. Le processus de rédaction, mis à la disposition de l'usager de Wikipédia par la «transparence " des pages de discussion, contribue à éclairer ce sujet. L'analyse des discussions montre en effet que la qualification d'une entrée comme neutre dépend plus du consensus des participants, de leur aspiration à ne pas basculer dans un dialogue de sourds, que de la neutralité réelle, linguistiquement parlant, et qui plus est, de la neutralité telle qu'elle est définie et exigée par Wikipédia. Plus la discussion est sereine et coopérative, plus il y a de chances qu'un commun accord soit atteint et inversement. Il y a ici une hiérarchisation différente des valeurs épistémiques qui opposerait Wikipédia aux encyclopédies d'autrefois pour lesquelles la vérité référentielle est absolutisée. Ceci est confirmé par la conception participative de la construction du « fait », qui est une des spécificités de la « neutralité » wikipédienne.

La nouveauté de la conception interactive que ce site représente dans la construction du savoir implique un changement dans la disposition des esprits à l'égard de la notion de neutralité. Alors que l'encyclopédie classique propose un ethos d'expertise et de finitude, Wikipédia par contre propose un ethos de souplesse infinie, puisque toujours ouverte au changement. Le savoir se construit par discussion participative et jamais terminée, ad infinitum. Le fait n'est pas absolu et il dépend de l'accord entre les contributeurs. Pour Wikipédia il s'agit donc d'une aspiration permanente à la neutralité plutôt que de l'affirmation péremptoire de pouvoir être neutre. Cette pratique suggère que de la même manière que nul ne détient la Vérité, la Justice ou la Rationalité absolues, nul ne peut prétendre adopter un point de vue absolument neutre. Il est tout de même possible et légitime d'aspirer à y arriver. La distance ${ }^{21}$ qui existe entre chaque point de vue individuel et la Vérité absolue peut être négociée entre les êtres humains à l'aide des outils de l'argumentation. Comme l'affirme Perelman, la rationalité et le point de vue de chacun nécessite la validation de l'Autre pour pouvoir arriver à un accord.

Mais cette pratique innovatrice de Wikipédia représente aussi, me semble-t-il, une conception plutôt relativiste du savoir : relative au consensus et à la convivialité entre 
les participants à la rédaction d'une définition. Il peut paraitre malgré tout inquiétant pour la conception de la construction du savoir que, lorsque les contributeurs votent pour ou contre la mention d'une entrée "de qualité » et «neutre ", l'aspiration à un processus de rédaction convivial et serein soit mise de facto au-dessus de l'examen critique de la présentation pluraliste des points de vue. Il semble que cette aspiration permanente à la neutralité et la responsabilité collective de l'acte de dire imposée par la nouvelle pratique de Wikipédia ne donne pas encore à l'utilisateur de l'Encyclopédie du $21^{\mathrm{e}}$ siècle la garantie que le savoir qu'il y consulte est effectivement le plus neutre possible.

\section{BIBLIOGRAPHIE}

Amossy, Ruth. 2011. «La coexistence dans le dissensus ", Semen 31, « Polémiques médiatiques et journalistiques », 25-42

Benveniste, Emile. 1966. « De la subjectivité dans le langage ", Problèmes de linguistique générale,2 vol. (Paris : Gallimard), 1 : 258- 266

Charaudeau, Patrick. 1997. Le discours d'information médiatique. La construction du miroir social (Paris : Nathan-INA)

D’Alembert, Jean le Rond. 1751. « Discours préliminaire des éditeurs », Encyclopedie ou Dictionnaire raisonné des sciences, des arts et des métiers (Paris : Le Breton), i-xiv

Dreyfus, Ferdinand-Camille. 1886. "Préface », La grande encyclopédie : inventaire raisonné des sciences, des lettres et des arts (Paris : édition H. Lamirault et $\mathrm{C}^{\mathrm{ie}}$ ), 1-9

Grand dictionnaire encyclopédique Larousse. 1982. « Avant-propos » (anonyme). (Paris : éditions Larousse)

Kerbrat-Orecchionni, Catherine. 2009 [1980]. L'énonciation: de la subjectivité dans le langage (Paris : Colin)

Kerbrat-Orecchioni, Catherine. 1981. «Argumentation et mauvaise foi », L'argumentation (Lyon: Presses universitaires de Lyon), 41-63

Koren, Roselyne. 1996. Les enjeux éthiques de l'écriture de presse et la mise en mots du Terrorisme (Paris : L'Harmattan)

Koren, Roselyne. 2004. «Argumentation, Enjeux et Pratiques de l'“engagement neutre" : le cas de l'écriture de presse », Semen 17, «Argumentation et prise de position : pratiques discursives », $19-40$

Langlais, Pierre-Carle. 2015. « \{\{Référence nécessaire\}\} L'émergence d'une norme wikipédienne (2003-2009) ", Barbe, Lionel, Louise Merzeau \& Valérie Schafer (éds). Wikipédia, objet scientifique non identifié (Paris : Presses universitaires de Paris Ouest), 77-90

Meyer, Michel. 2008. Principia Rhetorica. Une théorie générale de l'argumentation (Paris : Fayard)

Perelman, Chaïm. 2012 [1977]. L'empire rhétorique (Paris : Librairie philosophique Vrin) 
Perelman, Chaïm, Lucie Olbrechts-Tyteca. 2008 [1958]. Traité de l'argumentation (Bruxelles : Editions de l'Université de Bruxelles)

Plantin, Christian. 2002 « Analyse et critique du discours argumentatif », Amossy, Ruth, Roselyne Koren (éds). Après Perelman (Paris : L'Harmattan), 229-263

\section{Pages internet}

« Discussion :Lehi » (s. d.), Wikipédia - l'encyclopédie libre :http://fr.wikipedia.org/wiki/ Discussion:Lehi\#Divers (consulté le 29-01-2012)

« Lehi » (s. d.), Wikipédia - l'encyclopédie libre : http://fr.wikipedia.org/wiki/Lehi (consulté le 29-01-2012)

« Lehi (group)» (s. d.), Wikipedia-the free encyclopedia: http://en.wikipedia.org/wiki/Lehi_(group) (consulté le 10-12-2012)

« Wikipédia : Principes fondateurs » (s. d.), Wikipédia - l'encyclopédie libre: http://fr.wikipedia.org/ wiki/Wikip\%C3\%A9dia:Principes_fondateurs

(consulté le 13-06-2011)

«Wikipédia: Neutralité de point de vue » (s. d.), Wikipédia-l'encyclopédie libre: http://

fr.wikipedia.org/wiki/Wikip\%C3\%A9dia:Neutralit\%C3\%A9_de_point_de_vue (consulté le

21-08-2011)

Yahoo! Questions réponse (s. d), https://fr.answers.yahoo.com/question/index?

qid=20090107032529AAaP4nF (consulté le 22-12-2014)

\section{NOTES}

1. Cet article est la version remaniée d'une partie d'une thèse de Master 2 portant le même titre. Le corpus entier contient huit entrées comme détaillé dans la note 12 ci-dessous.

2. Jimmy Wales, le fondateur du site, exprime ce souhait en ces termes : «Imaginez un monde où chaque personne sur la planète peut accéder librement à la totalité du savoir humain.». Comparez par exemple avec d'Alembert et Diderot, rédacteurs de l'Encyclopédie: «le but d'une Encyclopédie est de rassembler les connaissances éparses sur la surface de la terre; d'en exposer le système général aux hommes avec qui nous vivons, \& de le transmettre aux hommes qui viendront après nous ".

3. Le travail lexicographique classique demande lui aussi du travail de coopération, dans la mesure où plusieurs experts de domaines différents rédigent des entrées concernant leurs domaines respectifs. Dans Wikipédia chaque entrée peut être rédigée par un groupe de plusieurs contributeurs qui vont et viennent, alors que dans l'encyclopédie classique le travail du rédacteur est la plupart du temps solitaire.

4. Une conception par ailleurs contestée, comme le fait remarquer Alain Rabatel (que je remercie de ses remarques) en citant Woody Allen: «L'objectivité à la télé ? Un quart d'heure pour les Juifs, un quart d'heure pour Hitler » (Woody Allen, Le Canard enchaîné, 07-05-2008).

5. Le discours préalable de La grande encyclopédie de 1886 reflète la recherche des mêmes valeurs : «[La Grande encyclopédie] expose les faits avec une scrupuleuse exactitude, les théories diverses ou contradictoires avec impartialité : il appartient au lecteur de comparer et de conclure. ». La " scrupuleuse exactitude" que valorise l'introduction de l'encyclopédie désigne une attitude éthique rigoureuse requise pour " exposer» le savoir dans l'ouvrage. Le travail d'exposition requiert donc de la part du lexicographe non seulement la prise en charge de la vérité 
référentielle de ses énoncés, mais aussi celle d'une éthique donnant la primauté au pluralisme et à l'équité.

6. Cf. Kerbrat-Orecchioni $2009: 179-186$, Mainguenau $2004: 190-195$, Koren $1996: 56$.

7. Une position similaire est reconnaissable dans l'introduction du Grand dictionnaire encyclopédique Larousse, édition de 1982 : «Les auteurs de l'ouvrage [...] se sont efforcés, dans les choix qu'implique le dictionnaire et dans la formulation des textes qui le constituent, de respecter les convictions de chacun. ». La formulation des textes des entrées demande donc un « effort » particulier de la part des rédacteurs pour éliminer de la mise en mots les points de vue individuels qui sont les leurs.

8. «Bien des notions en linguistique, peut-être même en psychologie, apparaîtront sous un jour différent si on les rétablit dans le cadre du discours, qui est la langue en tant qu'assumée par l'homme qui parle, et dans la condition d'intersubjectivité, qui seule rend possible la communication linguistique » (Benveniste, E., $1966: 266$ ).

9. «[...] dans l'argumentation, la notion de «fait » est caractérisée uniquement par l'idée que l'on a d'un certain genre d'accords au sujet de certaines données, celles qui se réfèrent à une réalité objective et qui désigneraient, en dernière analyse, pour citer H. Poincaré 'ce qui est commun à plusieurs êtres pensants et pourrait être commun à tous'. ». (Perelman et OlbrechtsTyteca 2008 [1958] : 89).

10. Il faut dire que l'idéal de neutralité n'a pas toujours été une norme incontournable de l'écriture encyclopédique. Au contraire, pour Diderot et d'Alembert elle était la faute à éviter. Selon Diderot, un bon dictionnaire doit « changer la façon commune de penser » (Article «Encyclopédie » de l'Encyclopédie, t. V : 635). En d'autres termes, l'héritage que Diderot lègue aux rédacteurs d'encyclopédies qui lui succèdent, est celui de l'esprit de combat qui en émane, combat contre le pouvoir, les autorités, le clergé. Diderot par ce choix illustre l'idée de Hobbes, qui dit que le pouvoir politique le plus fort appartient à celui qui détermine le sens des mots.

11. Voir annexe : «Introduction de l'entrée «Lehi », version du 29-01-2012)». Le Lehi était une des organisations clandestines juives à l'époque du mandat britannique en Palestine. Cette organisation, dirigée au début par Avraham (Yaïr) Stern, prônait, à la différence des autres organisations juives, le combat armé contre le pouvoir britannique.

12. Le corpus entier utilisé pour cette recherche contient huit entrées de Wikipédia (voir note 1). Elles concernent différents domaines de la vie sociale : médecine, économie et politique. Ce choix a été effectué d'après une évaluation intuitive du degré de polémicité de ces domaines. Dans chacun de ces domaines ont été retenues deux entrées : une entrée qui est accompagnée de la mention: "controverse de neutralité ", ce qui signifie qu'au moins l'un des contributeurs considère l'entrée comme non-neutre et une entrée qui est qualifiée d'«article de qualité ». Je choisis de présenter ici l'analyse de l'entrée considérée comme «neutre » dans le domaine du politique (« Lehi »).

13. Une comparaison avec le pendant non-neutre de l'entrée « Lehi » (Hamas) aurait pu procurer une image plus large et plus complète de la situation, mais le cadre de cet article ne nous le permet pas. Nous présumons néanmoins qu'il sera possible de saisir le processus de rédaction à partir d'une seule entrée.

14. L'identité des contributeurs n'est pas connue par l'utilisateur dans la majorité des cas, étant donné qu'ils signent avec un pseudonyme.

15. L'auteure recourt ici à la première personne afin de signaler qu'elle prend en charge l'énonciation d'un jugement de valeur concernant la rectitude éthique de l'introduction. Cette décision est due au fait que cette introduction semble allier description et prise de position. L'engagement éthique du chercheur poursuit en fait un but épistémique : s'abstenir de donner une partie cruciale des informations, c'est configurer un savoir incomplet. Il m'est cependant important de signaler que toutes les précautions possibles sont prises afin de présenter une analyse ancrée dans le discours, au ras des mots et qui justifiera mes décisions. 
16. Pour plus d'information concernant les différents types d'interventions, consultez KerbratOrecchionni (2009 [1980] : 135-162).

17. https://fr.answers.yahoo.com/question/index?qid=20090107032529AAaP4nF, consulté le 22-12-2014.

18. Patrick Charaudeau $(1997: 37,88)$ met en garde sur la fragilité des «effets » supposés des discours. Or, ce que nous présentons ici n'est pas "supposé », mais bien formulé dans des discours authentiques, circulant dans les « espaces publics » de l'interdiscours. Nous considérons donc ces interventions comme une possible preuve de ce que nous avançon plus haut.

19. Voir annexe «Extrait de la discussion concernant l'entrée "Lehi" ».

20. Voir notre explication concernant les principes fondateurs de Wikipédia dans le chapitre 1.

21. A la différence de Michel Meyer (2008), qui parle du concept de la « distance » pour exprimer la différence entre les positions de chacun des participants à la discussion, j'utilise ce concept pour définir la position de chacun des interlocuteurs par rapport à la « Vérité absolue ».

\section{RÉSUMÉS}

Cet article concerne la notion de neutralité dans le contexte de l'écriture encyclopédique du site Wikipédia. Le principe fondateur de Wikipédia intitulé La neutralité de point de vue atteste de l'aspiration du site à présenter une information fiable et dépourvue de tout biais idéologique - un principe qui exige une écriture équilibrée dans le cas d'opinions diverses ou opposées. En un premier temps, on analyse la mise en mots du principe de neutralité tel qu'il est conçu par Wikipédia, pour le définir et le confronter avec les postulats des linguistes de l'énonciation et des analystes du discours du siècle dernier. En un deuxième temps, l'analyse critique d'un extrait de l'entrée «Lehi » permet de s'interroger sur la conformité de la rédaction des entrées aux règles de l'encyclopédie. L'écart qui ressort de l'analyse soulève la question de savoir à quoi réfère en réalité le concept de «neutralité » dans les entrées que les contributeurs qualifient comme telles. L'examen de la discussion montre que dans le processus qui confère à une entrée la mention "article de qualité », à savoir entre autres «neutre», les valeurs de convivialité et de coopération entre les contributeurs l'emportent sur le devoir d'impartialité et de suspension de la prise de position.

This article deals with the notion of "neutrality" within the context of Wikipedia. One of the "five pillar" guidance principles for editors on the website is named the "Neutral point of view", reflecting the website's aspiration to present a trustworthy information devoid of any ideological bias - a principle calling for balance in cases of diverging or conflicting opinions. The article first analyzes the definition of the neutrality principle in Wikipedia in order to confront it with linguistic postulates and up-to-date discourse analysis theories. It then presents a critical analysis of a fragment of the entry "Lehi" in French in order to examine the conformity between the entry's phrasing and the rules on neutrality established by Wikipedia. The discrepancy between the two raises a question concerning the actual meaning of the concept of "neutrality" when it is used by contributors to qualify entries as such. French Wikipedia has a definition of an entry as "quality article", which means it is inter alia estimated as "neutral". An analysis of the discussion between the editors shows that in the process leading to the label "quality article", the values of conviviality and cooperation between the contributors outweigh the duty of impartiality and non-bias writing. 
INDEX

Mots-clés : analyse du discours, discours encyclopédique, neutralité, Wikipédia

Keywords : discourse analysis, encyclopedic discourse, neutrality, Wikipedia

\section{AUTEUR}

RIVKA DVIRA

Université Bar Ilan, ADARR 